\title{
Entre rhétorique et pratiques. L'éducation à la citoyenneté en Espagne
}

From rhetoric to practice. Citizenship education in Spain

Entre retóricas y prácticas. Educación ciudadana en España

\section{J. Ramón Jiménez Vicioso et Juan Carlos González Faraco}

\section{OpenEdition}

\section{Journals}

Édition électronique

URL : https://journals.openedition.org/ries/136

DOI : $10.4000 /$ ries. 136

ISSN : 2261-4265

Éditeur

France Education international

Édition imprimée

Date de publication : 1 avril 2007

Pagination : $45-54$

ISBN : 978-2-85420-569-5

ISSN : $1254-4590$

\section{Référence électronique}

J. Ramón Jiménez Vicioso et Juan Carlos González Faraco, « Entre rhétorique et pratiques.

L'éducation à la citoyenneté en Espagne », Revue internationale d'éducation de Sèvres [En ligne], 44 | avril 2007, mis en ligne le 23 juin 2011, consulté le 08 juillet 2021. URL : http://journals.openedition.org/ ries/136 ; DOI : https://doi.org/10.4000/ries.136 


\title{
Entre rhétorique et pratiques
}

\section{L'éducation à la citoyenneté en Espagne}

\author{
J. Ramón Jiménez Vicioso \\ J. Carlos González Faraco
}

\section{ÉLÈVES ET CITOYENS}

Tout au long de la modernité, l'École a été l'un des lieux fondamentaux de l'apprentissage de nouveaux savoirs et où se construisaient, en grande partie, l'enfance et la jeunesse. Si elle continue encore aujourd'hui à jouer ce rôle, elle doit désormais le faire dans le cadre d'un réseau complexe d'expériences non scolaires dont l'influence sur la socialisation est extraordinaire ${ }^{1}$. Ce sont ces nouvelles expériences, nées de la profonde restructuration qu'a subie la vie familiale et sociale ainsi que de l'accès des enfants aux langages technologiques et à certains espaces de loisir, qui articulent maintenant l'identité scolaire de l'enfant, c'est-à-dire sa condition d'élève, et non l'inverse. Cependant, ces changements dans lesquels baignent les enfants ne sont pas identiques partout (Hultqvist et Dahlberg, 2001), pas plus qu'ils ne suivent une règle culturelle unique; il n'est donc pas surprenant que règne aujourd'hui une certaine confusion sur la définition de l'«enfance» et, par conséquent, sur les spécificités souhaitables en matière de citoyenneté dans la société globale de ce nouveau millénaire.

À l'aube de l'ère moderne, sous l'impulsion des Lumières, sont apparus divers «mythes» autour de l'individualité et les États ont formé des plans ambitieux pour «fabriquer» de nouveaux citoyens, tels que les grands systèmes d'éducation générale (Hunter, 1994; Popkewitz, 1998). Aujourd'hui aussi, à l'ère de l'information, dans la société mondialisée de la modernité tardive, émergent de nouvelles icônes et de puissantes représentations de l'enfant, c'est-à-dire du futur citoyen. Il s'agit d'une nouvelle ère de l'enfance, une enfance postmoderne indissociable des médias de communication de masse et de l'inexorable développement d'une culture populaire par les grandes entreprises commerciales. Comme l'affirment J.-L. Kincheloe et S. R. Steinberg (2000, 30), "l'accès des enfants d'aujourd'hui à une culture enfantine commerciale et à la culture populaire ne les amène pas seulement à se convertir en consommateurs hédonistes, mais elle endommage aussi leur innocence et leur capacité à se protéger des tribulations de l'existence adulte, protection dont ils bénéficiaient (...) depuis les débuts de l'ère de la protection de l'enfance dans les années 1950 ».

1. Voir «L'élève aujourd'hui : façons d'apprendre», Revue internationale d'éducation de Sèvres, nº 29, mars 2002. 
Ces changements importants influencent leurs relations avec les adultes dont les espaces de connaissance, auparavant exclusifs, leur sont désormais facilement accessibles. Au fur et à mesure que les médias favorisent la prolifération infinie du sens, les limites entre l'enfance et l'âge adulte se dissipent; enfants et adultes sont confrontés au même paysage médiatique et se heurtent aux mêmes obstacles pour en extraire une signification. Les enfants s' «adultisent» et les adultes s' "infantilisent», écrit J. L. Kincheloe (2000, 58). Ces circonstances nouvelles ont fragilisé les traditionnelles bases conceptuelles, curriculaires et administratives sur lesquelles s'établissait la scolarisation. Que pouvons-nous faire? se demandaient Steinberg et Kincheloe devant ces changements radicaux de la culture enfantine. Les grands empires commerciaux, qui ont pour cibles principales les enfants, sont en train d'hériter, dans une certaine mesure, - voire de l'usurper, dans certains cas -, de l'ancienne prérogative de l'État comme «légitime administrateur des âmes» (Popkewitz, 1998) et se sont constitués en redoutables usines à fabriquer des désirs. Nous assistons, sans l'ombre d'un doute, à une reconstruction de l'enfance problématique, qui a déjà toute une série d'effets sur le rôle des adultes et de tous les acteurs sociaux impliqués dans l'éducation ainsi que, bien sûr, sur l'idée de citoyenneté.

Ces connexions sont spécialement évidentes dans un pays comme l'Espagne au système démocratique encore jeune. Dans presque tous les discours, la modernisation, la démocratie et l'éducation apparaissent comme étroitement liées entre elles, comme des faces de la même pièce de monnaie. En conséquence, «l'étudiant idéal» est toujours une représentation précoce du «citoyen idéal» dans les nombreuses réformes éducatives qui se sont succédé au cours des trente dernières années (Pereyra, González Faraco et Coronel, 2002). Dans la mesure où toute réforme éducative suggère un projet politique (et un modèle de citoyen), le débat - voire le conflit - naît immédiatement entre divers secteurs sociaux et idéologiques, divers groupes de pouvoir et acteurs éducatifs à différents niveaux. Il n'est de ce fait pas étonnant que dans toute l'Europe, et avec plus d'acuité encore en Espagne, l'éducation à la citoyenneté soit le terrain privilégié des discussions et des confrontations. Dans des temps si incertains, marqués par la perte évidente de points de repère communs et par des évolutions culturelles vertigineuses (Berger et Luckmann, 1995), tous reconnaissent la nécessité de promouvoir cette matière; mais son orientation, son contenu et son évaluation restent controversés et très problématiques.

L'éducation à la citoyenneté fait actuellement en Espagne l'objet d'un débat de fond qui dépasse largement la question des programmes et le domaine de l'école. Les discours officiels, les instructions officielles et les recommandations pédagogiques insistent sur la nécessité et même l'urgence d'une éducation qui prépare l'élève comme futur citoyen d'une société toujours plus mondialisée, complexe et multiculturelle dans laquelle certains perçoivent des risques pour la démocratie, la cohésion sociale et la vie en commun 
(Bartolomé, 2002; Bolivar, 2005; Imbernón, 2003). La question est de savoir si ce discours, omniprésent, a déjà sa place dans les salles de classe, s'il est en train de modifier, même a minima, la culture scolaire ou si, au contraire, il ne s'agit que d'une proposition rhétorique promise à peu d'avenir.

Malgré des convergences et des objectifs partagés, l'éducation à la citoyenneté est comprise de façon différente dans la tradition éducative de chaque pays européen et renvoie à l'actualité, parfois pressante, de chacun. Cet article essaiera d'approfondir le cas espagnol en considérant les textes et les discours d'un côté, et les pratiques scolaires, de l'autre.

\section{L’ÉDUCATION À LA CITOYENNETÉ DANS LA RÉFORME SOCIALISTE}

Comme nous l'avons dit, la construction de l'idée de citoyenneté à travers l'éducation est une préoccupation commune à tous les pays européens. Le Conseil de l'Europe - ainsi que d'autres organisations supranationales comme l'UNESCO (2005) - insiste depuis un certain temps déjà sur la nécessité de redéfinir la citoyenneté européenne et, en conséquence, d'initier une nouvelle approche éducative qui prenne en compte les nouvelles réalités du $\mathrm{XXI}^{\mathrm{e}}$ siècle et contribue à une plus grande cohésion sociale et politique. Un rapport d'EURYDICE (2005) propose certains axes de base dans ce domaine ${ }^{2}$ :

- le développement de la culture politique, qui inclurait la connaissance des institutions politiques, ainsi que les droits humains, les problèmes sociaux actuels, les constitutions nationales, la reconnaissance du patrimoine culturel et historique et la promotion de la diversité culturelle et linguistique de la société;

- le développement de la pensée critique et de certaines attitudes et valeurs telles que le respect de soi-même et des autres, l'esprit de solidarité, la résolution des conflits de façon pacifique ou le développement de stratégies pour combattre le racisme et la xénophobie;

- la participation active des élèves en leur offrant des expériences pratiques de démocratie dans l'établissement éducatif, en encourageant une plus grande implication dans les communautés locale, nationale et internationale, etc.

En Espagne comme dans d'autres pays de l'Union européenne, la question de l'éducation à la citoyenneté est depuis quelque temps associée à d'autres débats, ce qui représente, outre un enrichissement évident, une source inépuisable de difficultés pour son développement en tant que matière

\footnotetext{
2. L'étude menée par Eurydice en 2005 , L'éducation à la citoyenneté à l'école en Europe, offre une vaste analyse de cette question pour les pays de l'Union européenne. Ce document propose en outre une définition de certains concepts, des informations sur les programmes d'enseignement, une description des systèmes de participation à l'organisation démocratique dans les établissements d'enseignement, ainsi que quelques paramètres pour l'évaluation de l'éducation à la citoyenneté.
} 
d'enseignement. Il ne faut pas oublier que la «question scolaire» a toujours été en Espagne une affaire particulièrement sensible dans la politique nationale et que, en quinze ans, se sont succédé précipitamment trois réformes scolaires de grande envergure. La question des valeurs, le caractère laïque de l'éducation ou encore l'éducation civique ont suscité, ensemble ou séparément, de vives polémiques, principalement entre les groupes politiques conservateurs et progressistes, ainsi qu'entre l'église catholique et le gouvernement. Il convient aussi de se souvenir, pour bien comprendre la situation espagnole, que la structure décentralisée de l'État confie aux gouvernements régionaux (communautés autonomes) une grande partie des décisions en matière de politique éducative.

Comme nous l'avons déjà dit, toutes les réformes scolaires engagées en Espagne ont proposé un modèle de citoyenneté, chacune avec des caractéristiques quelque peu distinctes. Cependant, seule la dernière réforme impulsée par le gouvernement socialiste en 2006 mentionne explicitement que la scolarité obligatoire doit contribuer à la formation des citoyens. Dans les réformes antérieures, il fallait la "dénicher» dans des propositions pédagogiques beaucoup plus diffuses comme l' «éducation aux valeurs» ou les "axes transversaux du curriculum ${ }^{3}$. Aujourd'hui, dans la Loi organique de l'éducation (LOE 2006), sa présence est nette et soulignée, au point d'avoir été l'un des éléments les plus symboliques et les plus mis en avant de la réforme socialiste ${ }^{4}$.

De fait, l'éducation à la citoyenneté existe déjà dans les nouveaux programmes comme matière spécifique que doivent obligatoirement suivre tous les élèves à des étapes distinctes de la scolarité. Elle acquiert ainsi beaucoup plus de solidité, au niveau des programmes, que celle que lui conféraient les anciens «axes transversaux». La loi socialiste dit expressément (article 18) que "l'éducation à la citoyenneté et aux droits de l'homme» sera introduite dès le troisième cycle (élèves âgés de dix à douze ans) de l'école primaire et continuera dans le secondaire au niveau du collège, dans le cadre de la scolarité obligatoire (élèves âgés de douze à seize ans); elle recommande, dans les deux cas, une attention particulière au respect de l'égalité entre les sexes. En complément, les instructions officielles prévoient, à la dernière année du collège une matière similaire intitulée «éducation éthique et civique» et au lycée une matière également obligatoire pour tous les élèves, «philosophie citoyenne».

Les bases des programmes d'enseignement dans ces matières ont déjà été publiées et devront être adaptées par chaque communauté autonome pour son propre territoire. Dans le cas de l'éducation primaire (décret du ministère de

3. Nous appelons «axes transversaux » ces contenus d'apprentissage qui doivent être traités «transversalement » dans toutes les matières et situations éducatives. Nous parlons de l'éducation à la paix, de l'éducation à l'environnement, de l'éducation à la santé et à la consommation, de l'éducation interculturelle et d'autres domaines similaires. Sans écarter l'intérêt théorique de cette proposition pédagogique innovante, il est certain que dans la pratique scolaire, les "axes transversaux» sont transformés habituellement en objectifs de second rang face aux matières traditionnelles (mathématiques, langue, etc.) (BOLIVAR, 2005).

4. Loi organique d'éducation, 3 mai 2006. 
l'éducation du 7 décembre 2006), divers objectifs et compétences sont mentionnés et trois blocs de contenus ${ }^{5}$ ont été établis, que l'on peut résumer ainsi :

- individu et relations interpersonnelles et sociales : autonomie et responsabilité personnelle; valorisation de l'identité de chacun et de celle de tous les autres; dignité humaine; droits de l'enfant et droits de l'homme; différences de sexe et égalité entre hommes et femmes, etc.;

- vie en communauté : valeurs de la société démocratique (tolérance, justice, solidarité, coopération et culture de paix); application de ces valeurs civiques à des situations de conflit dans des contextes sociaux distincts (familial, scolaire, etc.) ; responsabilité dans l'exercice des droits et des devoirs sociaux; résolution dialoguée de conflits; respect de la diversité culturelle et religieuse; identification de situations de marginalisation et de discrimination; valorisation de la participation à la vie collective;

- vie en société : principes de la vie sociale en commun; connaissance, estime et respect des biens communs et des services publics; développement d'habitudes civiques et du service à la communauté (protection civile, sécurité, défense, etc.); respect du code de la route. L'organisation pédagogique prévue pour ces contenus est cyclique, c'est-à-dire qu'ils seront abordés de façon répétée à chaque niveau mais avec un approfondissement différent. Ainsi, alors que, au primaire, on insistera sur la participation active des élèves à des instances plus proches, comme par exemple les conseils d'enfants au niveau de la classe, on analysera, au collège et au lycée, la participation politique dans les sociétés démocratiques.

\section{LE dÉBAT POLITIQUe, SOCIAL ET ÉDUCATIF}

Malgré un accord général sur la nécessité d'améliorer la formation citoyenne des élèves espagnols, il n'y a pas eu de consensus autour de cette proposition socialiste de faire de l'éducation à la citoyenneté une matière obligatoire aux contenus déterminés. Au contraire, cela a suscité un certain débat social et un affrontement beaucoup plus idéologique et politique que pédagogique du fait que, dès le début, on a fait le lien - et comparé - avec la traditionnelle matière «religion catholique» en présentant parfois ces deux matières comme antagonistes.

Comme nous l'avons indiqué dans d'autres textes ${ }^{6}$, l'une des controverses récurrentes en matière d'éducation est celle qui porte sur son caractère

5. Dans tous les contenus se répète l'attention particulière que méritent les questions relatives à l'égalité des sexes, ce qui reflète l'importance sociale et politique croissante prise par cette question. Pendant la phase de préparation de la réforme, il avait même été envisagé de créer une matière spécifiquement destinée à encourager l'égalité hommes - femmes, option qui fut finalement écartée.

6. Voir Jiménez Vicioso et Jiménez García, 2006, et aussi González Faraco, 2006. 
laïque ou confessionnel. La Constitution espagnole - rédigée à la fin des années soixante-dix durant une transition politique délicate entre la dictature franquiste et le nouveau régime démocratique - est hautement ambiguë sur cette question dans la mesure où, à chaque fois qu'une réforme scolaire est engagée, se rallume la querelle entre l'Église et l'État. D'autre part, l'introduction de l'éducation à la citoyenneté est parallèle à une réduction visible de la présence de la religion catholique dans les écoles publique ainsi que de son importance dans l'enseignement. La hiérarchie ecclésiastique voit dans ce processus, initié par un parti de gauche, un pas de plus vers la laïcité scolaire. Dans ce contexte, certaines franges de l'Église considèrent qu'à travers l'éducation à la citoyenneté, l'État essaie d'introduire des valeurs laïques dans l'éducation et que de ce fait, elle devrait recevoir le même traitement que celui qui est accordé à l'éducation religieuse, c'est-à-dire être une matière laissée au choix des familles.

Certaines questions, initialement incluses dans les contenus de l'éducation à la citoyenneté, ont été ouvertement critiquées et refusées par l'Église et par certains conservateurs : concrètement, celles qui faisaient allusion à la pluralité des modèles familiaux dans la société contemporaine - ce qui inclut en Espagne les familles formées par des couples homosexuels. Cela a contribué à renforcer davantage la résistance de l'Église à cette nouvelle matière. À certains moments, la polémique a atteint un tel degré d'hostilité que certains évêques ont encouragé les pères catholiques à la "désobéissance civile» et à «l'objection de conscience", ce qui signifie que leurs enfants pourraient quitter l'école pendant le temps qui est consacré à cette matière. Le réseau des établissements confessionnels dépendant de l'Église catholique a adopté, en revanche, une attitude plus modérée en élaborant ses propres manuels scolaires qui adapteront, ou tout simplement supprimeront, les thèmes jugés plus problématiques du point de vue de la doctrine catholique.

Face à ces positions se trouvent ceux qui soulignent l'urgence d'une éducation à la citoyenneté devant les rapides et brusques changements sociaux survenus en Espagne au cours de ces dernières années (Gimeno Sacristán, 2001 ; Bartolomé, 2002; Martínez Bonafé, 2003), parmi lesquels on peut citer en particulier : une société espagnole de plus en plus multiculturelle en raison d'une immigration récente très importante; la transformation de la vie familiale et la persistance lamentable des inégalités entre les sexes ainsi que des violences sur les femmes; des problèmes d'environnement qui exigent une nouvelle perspective de développement; la nécessité de promouvoir auprès des jeunes l'engagement civique, la participation politique et l'exercice des droits et des devoirs du citoyen.

D'un point de vue plus proprement scolaire, l'éducation à la citoyenneté est aussi nécessaire pour affronter le problème du climat social dans les établissements scolaires. L'augmentation des problèmes liés à la vie en commun dans les classes est inquiétante : mauvais traitements entre camarades, perte 
d'autorité du corps enseignant, vandalisme et détérioration des installations scolaires, etc. Dans cette perspective, l'éducation à la citoyenneté acquiert un caractère essentiellement préventif des conduites antisociales chez les adolescents et les jeunes. Bien sûr, certains s'alarment de la rhétorique qu'impliquent ces discours et défendent une éducation à la citoyenneté inondant la totalité des pratiques scolaires et de la vie en commun dans les établissements scolaires. Un psychologue espagnol connu, Juan Delval (2006 : 12), expert en éducation, l'exprime ainsi : "ces changements ne peuvent s'obtenir en introduisant une nouvelle matière, l'éducation à la citoyenneté, ni en sermonnant les élèves sur la nécessité d'être sages, de coopérer et de s'intéresser au travail scolaire, pas plus qu'en leur répétant qu'ils doivent faire davantage d'efforts ou en essayant de leur imposer plus d'épreuves, d'examens ou d'obstacles. Au contraire, il est nécessaire de changer le climat et l'organisation des établissements scolaires, de prêter plus d'attention à la vie sociale, de laisser participer les élèves et de les impliquer en profondeur dans les activités qu'ils réalisent, pour qu'ils parviennent à devenir des citoyens autonomes.»

En effet, comme l'enseignait John Dewey il y a de nombreuses années (1916), on ne peut obtenir une bonne éducation citoyenne dans un système démocratique qu'en faisant l'expérience depuis l'enfance des principes et des pratiques de la citoyenneté démocratique et non en la considérant comme une simple préparation formelle à la participation du futur adulte au système démocratique. Selon Delval, pour que l'éducation à la citoyenneté cesse d'être seulement un beau discours, les écoles doivent procéder à des transformations radicales dans trois domaines fondamentaux :

- l'organisation sociale : en donnant une plus grande responsabilité à l'élève dans l'élaboration du règlement, dans l'analyse et la résolution des conflits, en étant extrêmement scrupuleux dans l'administration des sanctions et en constituant l'école, en somme, comme exemple de vie démocratique;

- les contenus d'apprentissage : en encourageant l'esprit critique à travers des contenus plus rationnels, en introduisant les élèves à une approche analytique des phénomènes sociaux, en cultivant les sciences sociales et historiques, en intégrant dans le curriculum l'étude des phénomènes propres à la «nouvelle citoyenneté» tels que le développement durable ou le multiculturalisme, et en essayant de faire en sorte que ces enseignements aient une plus grande proximité et une meilleure répercussion sur la vie personnelle et sociale des élèves;

- les relations de l'école avec la communauté : en ouvrant les établissements au contexte social et à ses problèmes, en ouvrant les programmes à tout ce qui intéresse et préoccupe les adolescents et les jeunes, comme par exemple l'analyse des causes et des conséquences des phénomènes de bandes de jeunes et de leur violence, ou l'influence des drogues sur la santé physique et mentale. 
Sans rejeter l'intérêt d'une matière spécifique, Delval défend, comme nous, l'idée que l'éducation à la citoyenneté est la colonne vertébrale des principes qui animent la vie des établissements, leur mode d'organisation, leur administration et l'ensemble des pratiques scolaires. Et la seule voie possible pour y parvenir, c'est de doter ce champ éducatif d'un caractère profondément expérimental.

\section{Paradoxes et atTEnTES DANS LA VIE DES ÉCOLES}

Paradoxalement, bien des raisons qui justifient une bonne éducation à la citoyenneté sont vécues dans les écoles comme des obstacles à son développement. À cet égard, certains drames survenus dans des établissements scolaires et abondamment commentés par les médias ${ }^{7}$ ont valeur d'exemple. Loin de renforcer la nécessité d'améliorer l'éducation à la citoyenneté comme expérience éducative quotidienne dans les classes, ces cas ont fait naître une certaine panique et, par voie de conséquence, une recherche presque obsessionnelle de solutions rapides et drastiques. Le durcissement des lois sur les mineurs, l'accroissement des mesures de contrôle dans les établissements scolaires (installation de caméras, présence de la police aux portes des collèges, etc.) ou l'augmentation de l'autorité du corps enseignant sont, entre autres, quelquesunes de ces mesures urgentes - en majorité plus disciplinaires et punitives que préventives -, qui commencent à être prises dans certains établissements scolaires espagnols.

Évidemment, nous ne devons ni minimiser ni occulter les effets dévastateurs sur l'image de l'école que cause la combinaison d'un accroissement réel des difficultés à vivre ensemble, surtout dans les collèges, et d'une préoccupation grandissante de l'opinion publique, visiblement conditionnée par la grande diffusion médiatique de ces problèmes. Des incidents qui, autrefois, ne passaient pas les murs de l'école et étaient considérés seulement comme "des affaires de gosses", attirent maintenant l'attention de la télévision aux heures de grande audience ou remplissent les pages d'Internet, soit parce que leur dénonciation publique se multiplie, soit parce que les agresseurs les exhibent sans le moindre scrupule sur le réseau virtuel.

Dans l'imaginaire collectif transparaît la sensation que les collèges et les lycées sont des lieux dangereux, que la maltraitance entre les élèves est normale et que les professeurs se sentent impuissants devant ces problèmes, pendant que les autorités restent impassibles ou se montrent incapables de les affronter.

7. Rappelons, à titre d'exemple, le cas de Jokin, un enfant de quatorze ans qui se suicida en 2004 après avoir subi de nombreuses humiliations et brimades de la part de ses camarades de lycée, et rencontré une certaine passivité du corps enseignant. Ce cas eut une répercussion extraordinaire dans les médias. 
Certains suggèrent, à travers une critique politique plus globale, que ce mal-être et ce «chaos» dans les salles de classe est finalement la conséquence d'erreurs politiques plus générales comme une excessive permissivité envers les jeunes ou la mise en œuvre de certaines "expériences sociales», comme les nouvelles formes de mariage et de famille, ou certaines mesures «démagogiques» dans les établissements qui concèderaient trop de pouvoir aux étudiants, etc.

En conséquence, la situation sociale révèle un grave dilemme. Pour certains, il convient de penser sur le long terme en favorisant des mesures fondamentalement éducatives (ce qui supposerait de favoriser la participation et la responsabilité des élèves et d'augmenter le degré de démocratie dans la vie des établissements). Pour d'autres, en revanche, il faut agir immédiatement afin de maîtriser, le plus vite possible, les problèmes de désordre et de dégradation de la vie en commun grâce à des mesures disciplinaires et à la récupération du pouvoir par les adultes, même s'il faut pour cela aller jusqu'à suspendre les droits acquis par l'ensemble des élèves tels que l'entretien préalable à la sanction ou le droit d'être jugé par des organes ou des personnes impartiales dans le cas d'un conflit. C'est de la résolution de ce dilemme, et d'autres, que dépendra la viabilité d'une éducation effective à la citoyenneté.

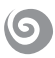

\section{3}

Il faut impérativement dépasser la rhétorique des discours qui sont, dans de nombreux cas, conditionnés par des divergences politiques et idéologiques plus générales. Un effort est nécessaire pour expliquer à l'opinion publique, et particulièrement à la communauté éducative - vraie protagoniste de la vie scolaire -, les virtualités et les possibilités de ce modèle d'éducation à la citoyenneté que propose la réforme actuelle. Il est sans doute indispensable de développer au sein des établissements un sentiment de communauté, à travers des actions de responsabilité partagée et de participation, à la fois pour aborder les problèmes quotidiens que pose la cohabitation et leur trouver des solutions - en valorisant et promouvant la solidarité (Gramigna, 2004) - et pour doter les futurs citoyens d'une formation solide dans le but d'une véritable pratique démocratique. 


\section{BibLIOGRAPHIE}

BARTOLOMÉ M. (Coord.) (2002) : Identidad y ciudadanía. Un reto a la educación intercultural. Madrid, Narcea.

BERGER P. L. et LUCKMANN T. (1995) : Pluralismus und Sinnkrise. Die Orienterung des Modernen Menschen. Gütersloh, Verlarg Bertelsmann Stiftung.

BOLÍVAR A. (2005) : «La Educación para la Ciudadanía en la agenda de las reformas». Pátio (Brasil), núm. 36 (noviembre), 12-15.

DELVAL J. (2006) : Hacia una escuela ciudadana. Madrid, Morata.

DEWEY J. (1995 [1916]) : Democracia y educación. Introducción a la filosofía de la educación. Madrid, Morata.

GIMENO SACRISTÁN J. (2001) : Educar y convivir en la cultura global. Las exigencias de la ciudadanía. Madrid, Morata.

GONZÁLEZ FARACO J. C. (2006) : «Les discours politiques sur l'enseignement religieux dans les écoles publiques espagnoles : les nouveaux aspects caractéristiques d'un vieux conflit». Education comparée, n 61 . Louvain-La-Neuve, Editions l'AFEC.

GRAMIGNA A. (2004) : «I fondamenti di una Pedagogia solidale nelle questioni sociali. Per una ermeneutica della prassi democratica». Dans ESCOLANO A. et GRAMIGNA A. Formazione e interpretazione. Milano, FrancoAngeli, pp. 129-150.

HULTQVisT K. et DAHLBERG G. (eds.) (2001) : Governing the Child in the New Millennium. New York, RoutledgeFalmer.

HUNTER I. (1994) : Rethinking the School. Allen \& Un win Pty Ltd.

IMBERNÓN F. (Coord.) (2003) : Cinco ciudadanías para una nueva educación. Barcelona, Graó.

JIMÉNEZ VICIOSO J. R. et JIMÉNEZ GARCÍA A. (2006) : «Le débat médiatique sur la laïcité en France et ses répercussions sur la presse écrite espagnole». Education comparée, no 61, pp. 213-225, Louvain-La-Neuve, Editions l'AFEC.

KINCHELOE S. R. (2000) : «Solo en casa y Malo hasta la médula : surge una infancia postmoderna». Dans STEINBERG S. R. et KINCHELOE J. L. (comps.) : Cultura infantil y multinacionales. Madrid, Morata, pp. 45-64.

MARTÍNEZ BONAFÉ J. (Coord.) (2003) : Ciudadanía, poder y educación. Barcelona, Graó.

PEREYRA M., GONZÁlez FARACO J. C. y CORONEL J. M. (Coord.) (2002): Infancia y escolarización en la modernidad tardía. Madrid, Akal.

POPKEWITZ T. S. (1998) : Struggling for the Soul. New York, Teachers College, Columbia University.

UNESCO (2005) : Educación para la ciudadanía. Aprender a vivir con los demás, respetándolos. Éste es el desafío de la educación para la ciudadanía. UNESCO. Paris. 